Unfallchirurg 2016 · 119:176

DOI 10.1007/s00113-016-0151-y

Online publiziert: 15. Februar 2016

(c) Springer-Verlag Berlin Heidelberg 2016

CrossMark

\author{
P. Biberthaler \\ Klinik und Poliklinik für Unfallchirurgie, Klinikum rechts der Isar, \\ Technische Universität München, München, Deutschland
}

\title{
Periprothetische Frakturen Teil 1
}

Sehr geehrte Leserinnen und Leser,

die Relevanz periprothetischer Frakturen nimmt exponentiell zu. Dies liegt zum einen begründet in der steigenden Implantationszahl von Endoprothesen. Diese Entwicklung liegt der stetig steigenden Lebenserwartung in den westlichen Industrienationen zugrunde. Die Menschen werden nicht nur immer älter, sie möchten auch ein Leben in größtmöglicher Lebensqualität und damit Mobilität führen. Somit steigt auch der funktionelle Anspruch kontinuierlich an. Die New Yorker Großmutter, die auf der Harley die Route 66 hinunter knattert, ist zum Sinnbild dieser Entwicklung geworden und steht zu einem gewissen Grad auch für den Erfolg der Unfallchirurgie und Orthopädie, deren herausragende therapeutische Entwicklungen der vergangenen Jahrzehnte es zumindest $\mathrm{zu}$ einem gewissen Teil den Menschen erst möglich gemacht hat, diese Mobilität zu leben. Somit ist es auch völlig klar, dass die Relevanz der Frakturen bei einliegenden Prothesen deutlich zunimmt, und hier muss das therapeutische Ziel sein, die Mobilität, die vor der Verletzung bestanden hat, so optimal wie möglich wieder herzustellen. Daher bin ich dem Springer-Verlag sehr dankbar, dass aufgrund dieser deutlich zunehmenden Relevanz das Thema zum Leitthema erhoben wurde und aufgrund der Menge der hierfür relevanten Informationen in 2 Heften dargestellt werden soll. Im ersten Band werden daher Prinzipien des Managements erklärt, Klassifikationssysteme an Hüfte und Knie erläutert und das Rehabilitationsmanagement dargelegt. Im zweiten Heft werden Prinzipien der oberen Extremitäten, der Revisionsendoprothesen und Osteosynthesen gezeigt. Ich freue mich sehr, dass es gelungen ist, namhafte Autoren zu überzeugen, aus ihrer großen Expertise zu diesem Thema, ausgedrückt in wegweisenden internationalen Publikationen, zu berichten und das Thema dem Leser der Zeitschrift Der Unfallchirurg vorzulegen.

Dies ist ein weiteres schönes Beispiel dafür, dass die Zeitschrift Der Unfallchirurg das Sprachrohr für aktuelle Entwicklungen in der deutschsprachige Orthopädie und Unfallchirurgie ist.

So wünsche ich Ihnen interessante Erkenntnisse im Sinne Ihrer Patienten und verbleibe mit herzlichen Grüßen

Ihr

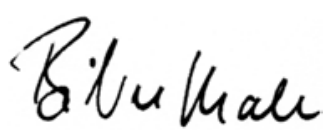

Prof. Dr. Peter Biberthaler

\section{Korrespondenzadresse}

Prof. Dr. P. Biberthaler
Klinik und Poliklinik für
Unfallchirurgie,
Klinikum rechts
der Isar, Technische
Universität München
Ismaningerstr. 22, 81675
München
Peter.Biberthaler@mri.tum.de

Interessenkonflikt. P. Biberthaler gibt an, dass kein Interessenkonflikt besteht. 